\title{
Simulation of high precision angle control in mechanical manufacturing Numerical Control Technology
}

\author{
Chen Jinxia \\ Inner Mongolia Technical College Of Mechanics And Electrics \\ 010070
}

Keywords: PID fuzzy control; angle detection; angle control;

\begin{abstract}
: during the study process high precision angle control in the mechanical manufacturing computer numerical control (CNC) technology, using the current algorithm to control the high precision angle of mechanical manufacturing is unable to realize the smoothing angle control, and angle control error is large. For this purpose, a high precision angle control method based on the improved PID fuzzy algorithm is proposed for mechanical manufacturing CNC technology. With the method, the angle detection system detects the signal and sends to the microcontroller, technology processing like scale conversion, digital filtering are followed in microcontroller. The treated measurement value and set value are compared, through digital PID computing to input digital control signal. On the basis of this, single input and single output nonlinear system equations of angle control are built to design an angle feedback controller based on fuzzy logical, the output of the system in a certain extent tracks and approaches ideal angle output of high precision, and complete high precision angle control in the machinery manufacturing CNC technology effectively. The experimental results show that the accuracy of high precision angle control method based on the improved PID fuzzy algorithm in mechanical manufacturing CNC technology is high, and the robustness is strong.
\end{abstract}

\section{Introduction}

In modern manufacturing industry, using a large number of computer numerical control (CNC) machine tools to replace the ordinary machine tools for high precision, high risk, high speed complex machining, super precision machining accuracy index has been increased to submicron ${ }^{[1-3]}$. With the continuous growth of high precision and complex surface parts processing, high precision angle has become an important precision target of the CNC system ${ }^{[4-6]}$. And high precision angle control technology in machinery manufacturing CNC technology is the main way to achieve the index, receive the extensive attention ${ }^{[7]}$, at the same time, there are a lot of good method ${ }^{[8-10]}$.

At present, the high precision angle control technology is mainly based on the new 5 degree of freedom parallel algorithm, fuzzy feedback algorithm and variable gain cross coupling control algorithm. Among them, the high precision angle control method based on the variable gain cross coupling control algorithm is commonly used. But the algorithm cannot achieve the smooth control of angle, angle control error is large.

In view of the above problems, a high precision angle control method based on the improved PID fuzzy algorithm is proposed for mechanical manufacturing CNC technology. The method has high accuracy and robustness.

\section{2 high precision angle control principle in mechanical manufacturing CNC Technology}

In high precision angle control process of machinery manufacturing CNC technology, according to the feedback information and the interpolation values of each axis, correct the gain of angle error model in real-time to find the optimal compensation law and send compensation correction information back to the shaft, so as to achieve the purpose to compensate the angle error, angle controller is defined as the combination of low-level controller and high-level controller. Specific steps are as follows:

In high precision angle control process of machinery manufacturing CNC technology, the angle error model can be obtained by using the following formula:

$$
X=-E_{x} \sin \theta+E_{y} \cos \theta
$$

In the formula, satisfied with the condition of $\sin \theta=V_{Y} / Y$.

In high precision angle control process of machinery manufacturing CNC technology, it is 
assumed that the conditions of $C_{x}=V_{y} / V$ and $C_{y}=V_{x} / V$ are set, the following equation is set: $X=-E_{x} C_{x}+E_{y} C_{y}$

On the basis of the formula, the angular error depends on the feed rate of each axis.

In high precision angle control process, and input variables of the angle controller are tracking error $x, y$ axis, and thus constitute a real-time error model of system, the output of controller is to provide additional effect amount of each axis.

\section{3 the optimization principle of high precision angle control in CNC technology of mechanical manufacturing}

In view of the current algorithm for mechanical manufacturing high-precision angle control, it cannot achieve the smooth control of angle, there is a large angle control error. A high precision angle control method based on the improved PID fuzzy algorithm in mechanical manufacturing NC technology is proposed.

\section{1 optimization measurement of angle}

In high precision angle control process, the angle detection system detects the signal and sends to the microcontroller, technology processing like scale conversion, digital filtering are followed in microcontroller. The treated measurement value and set value are compared, through digital PID computing to input digital control signal. Specific steps are as follows:

In the process of high precision angle control, the angle detection system detects the signal and sends to the microcontroller, technology processing like scale conversion, digital filtering are followed in microcontroller:

$\bar{Y}=\frac{1}{N} \sum_{i=1}^{N} y_{i}$

In the process of high precision angle control, the two adjacent angle sampling subtract to obtain increment of angle, the absolute value of angle increment and the maximum difference $\alpha$ allowed by two point sampling are compared. $\alpha$ size is decided by measured angle, if less than or equal to the allowable maximum difference, the sampling is effective, otherwise the last angle sampling is regarded as a sample of the data of this time. Using the following formula:

$\Delta y_{n}=\left|y_{n}-y_{n-1}\right|\left\{\begin{array}{l}\leq \alpha \bar{y}_{n}=\bar{y}_{n} \\ \geq a \bar{y}_{n}=\bar{y}_{n-1}\end{array}\right.$

In the process of high precision angle control, according to the above formula, the filtering result is one of the sample value of actual angle, and reflect the size of the measured angle authentically.

In the process of high precision angle control, the measured value is compared with the setting value, and the digital control signal is input after calculating by digital PID. Using the following formula:

$y=k_{p}\left[e(t)+\frac{1}{T_{I}} \int e(t) d t+T_{D} \frac{d e(t)}{d t}\right]$

In the process of high precision angle control in mechanical manufacturing numerical control technology, the upper equation is discretized and the lower equation is obtained:

$p_{n}=k_{e}\left[e_{n}+\frac{T_{S}}{T_{d}} \sum_{i=0}^{n} e_{i}+\frac{T_{d}}{T_{s}}\left(e_{n}-e_{n-1}\right)\right]$

In the upper equation, $p_{n}$ represents computer output value of $n$-th sampling, $e(n)$ is deviation value of $\quad n$-th sampling, satisfied with the condition of $e(n)=X-y_{n}$.

In the process of high precision angle control, the angle increment digital PID control law can be obtained:

$\Delta P_{n}=p_{n}-p_{n-1}$ 
3.2 the realization of the optimization principle of the high precision angle control in the mechanical manufacturing CNC technology

In the process of high precision angle control, the single input and single output nonlinear system equations of angel control are built with the following equation:

$y^{(n)}=f\left(x, \dot{x}, \ldots, x^{(n-1)}\right)+g\left(x, \dot{x}, \ldots, x^{(n-1)}\right) u$

In the formula, $f$ and $g$ are unknown nonlinear functions.

In the process of high precision angle control, an angle feedback controller based on fuzzy logic is designed:

$u=u(x \mid \alpha, \beta)$

In the process of high precision angle control, the output of the system is utilized to track and approach to the output of the system $y_{m}(t)$ to a certain extent, and satisfied with the following conditions:

$x=\left(x, \dot{x}, \ldots, x^{(n-1)}\right)^{T}$

$e=y_{m}-y \quad(11)$

$e=\left(e, \dot{e}, \ldots, e^{(n-1)}\right)^{T}$

In the process of high precision angle control, the control law of angle can be obtained by using the following formula:

$\stackrel{*}{u}=\frac{1}{g(x)}\left[-f(x)+k^{T} e+y_{m}^{(n)}(t)\right]$

In the process of high precision angle control, the fuzzy system is used to approximate $f(x)$ and $g(x)$ :

$u=\frac{1}{\tilde{g}\left(x \mid \theta_{g}\right)}\left[-\tilde{f}\left(x \mid \theta_{f}\right)+k^{T} e+y_{m}^{(n)}(t)\right]$

\section{4 experimental simulation}

In order to prove the proposed high precision angle control method based on improved fuzzy PID algorithm for mechanical manufacturing CNC technology is effective, a single experiment is needed, under the windows, $X P$ environment to build high precision angle control experiment simulation platform. The improved algorithm and the traditional algorithm for high precision angle control were used respectively, the angle control accuracy result of different algorithms are compared with high accuracy angle in need, and used to measure the effectiveness of high precision angle control of different algorithms, comparison results are shown in Figure 1.

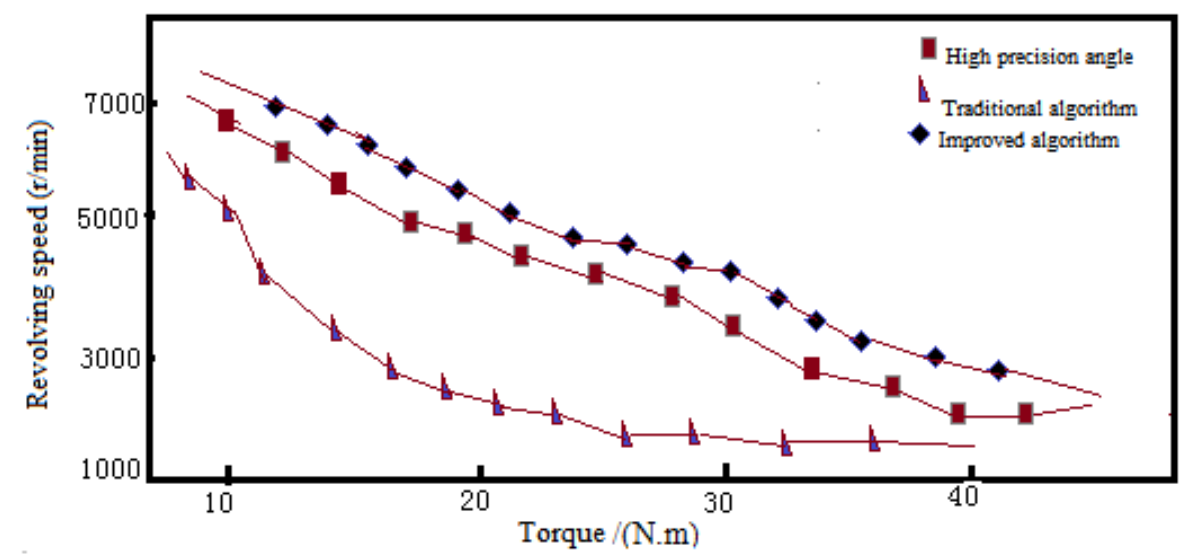

Fig. 1 Comparison of angle under different control modes

From table 1 it can be seen that the angle control based on the improved PID fuzzy algorithm is better than the traditional algorithm. The control accuracy is higher, and the robustness is stronger. 
This is because the angle detection system detects the signal and sends to the microcontroller, technology processing like scale conversion, digital filtering are followed in microcontroller. The treated measurement value and set value are compared, through digital PID computing to input digital control signal. On the basis of this, single input and single output nonlinear system equations of angle control are built to design an angle feedback controller based on fuzzy logical, the output of the system in a certain extent tracks and approaches ideal angle output of high precision, and complete high precision angle control in the machinery manufacturing CNC technology effectively.

\section{Conclusion}

The current algorithm used to control the high precision angle of mechanical manufacturing is unable to realize the smoothing angle control, and angle control error is large. For this purpose, a high precision angle control method based on the improved PID fuzzy algorithm is proposed for mechanical manufacturing CNC technology. With the method, the angle detection system detects the signal and sends to the microcontroller, technology processing like scale conversion, digital filtering are followed in microcontroller. The treated measurement value and set value are compared, through digital PID computing to input digital control signal. On the basis of this, single input and single output nonlinear system equations of angle control are built to design an angle feedback controller based on fuzzy logical, the output of the system in a certain extent tracks and approaches ideal angle output of high precision, and complete high precision angle control in the machinery manufacturing CNC technology effectively. The experimental results show that the accuracy of high precision angle control method based on the improved PID fuzzy algorithm in mechanical manufacturing CNC technology is high, and the robustness is strong.

\section{References}

[1] Liu Jiushan, Sun Xuguang, Ma Shuai. CCNC technology in mechanical manufacturing [J]. Chinese Technology Expo, 2013, (19): 22-22.

[2] Shi Yinhua. Application of CCNC technology in mechanical manufacturing [J]. Guide of Sci-tech Magazine, 2013, (33): 260-260.

[3] Jin Daxing. The application of digital technology in mechanical manufacturing [J]. Digital Technology and Application, 2013, (10): 220-220.

[4] Zhou Libo, Hu Gang. Study on the application of CCNC technology in mechanical manufacturing [J]. ScieCNCe and Technology Innovation Herald, 2014, 11 (6): 31-31.

[5] Wu Chaoyang. Analysis of the application of numerical control technology in mechanical manufacturing [J]. Guide of Sci-tech Magazine, 2013, (21): 334-334.

[6] Li Xiuchang. Discussion on the application of numerical control technology in the mechanical manufacturing [J]. Guide of Sci-tech Magazine, 2013, (9): 201-201.

[7] Chen Zhonghui. Analysis of the effective use of CCNC technology in mechanical manufacturing

[J]. Guide of Sci-tech Magazine, 2013, (2): 181-181.

[8] Jin Jianguo. Development and Application of CCNC in Mechanical Manufacture [J]. Technology and innovation, 2014, (22): 40-40.

[9] Wang Jing. Discussion on the use of technology in mechanical manufacturing, [J]. ScieCNCe \& Technology Vision, 2014, (18): 63-63.

[10] Miao Di, Liu Zhenjuan, Li Hongguang. Approach to Fuzzy Model-Free Adaptive Coordinating Control for Distillation Processes [J]. Computer simulation, 2013, 30 (1): 377-381. 